\section{Regionalização dos serviços de saúde: desafios para o caso de Angola}

\author{
Regionalization of health services: challenges for \\ the Angolan case
}

\footnotetext{
${ }^{1}$ Escola Nacional de Saúde Pública Sergio Arouca, Fundação Oswaldo Cruz, Rio de Janeiro, Brasil.

Correspondência E. Artmann Departamento de Administração e Planejamento em Saúde, Escola Nacional de Saúde Pública Sergio Arouca, Fundação Oswaldo Cruz. Rua Leopoldo Bulhões 1480 Rio de Janeiro, $R J$ 21041-210, Brasil. artmann@ensp.fiocruz.br
}

\begin{abstract}
This article presents the health situation in Angola from 2000 to 2007, discussing regionalization of the health system as an important instrument for improving access and the use of health services in the country, besides highlighting the conditions and challenges for implementation. The article is based on a literature review of: (a) Brazilian authors, considering that health reform is still under way in Brazil, with extensive academic output on this particular theme, (b) African authors or studies on Africa and international studies, considering the need to review regional and global experiences related to the subject, and (c) World Health Organization studies and guidelines on health decentralization and regionalization. The need for reform in the Angolan health system has been demonstrated by the enormous structural and operational deficiencies, leading in turn to low effectiveness and reliability. Regionalization was considered a key strategy for restructuring the health system. However, the evaluation of its impact will only be possible in the long term.
\end{abstract}

Health Services Accessibility; Regional Health Planning; Health Systems
Miguel dos Santos de Oliveira 1

Elizabeth Artmann 1

\section{Introdução}

Em muitos países africanos, apesar dos esforços que estes têm envidado para levar a saúde a todos os indivíduos, verificam-se enormes dificuldades na garantia do acesso de numerosos grupos populacionais aos serviços de saúde. Isto se deve à fragilidade organizacional dos sistemas de saúde, à crise econômica, à atenção inadequada aos princípios dos cuidados primários da saúde, à escassez dos recursos de toda ordem (financeiros, humanos, tecnológicos, entre outros) e/ou sua má distribuição 1 . Observam-se, ainda, o surgimento e o alastramento de epidemias como o HIV/AIDS, a excessiva concentração de recursos nos grandes centros urbanos, os conflitos civis, bem como a falta, em alguns casos, de uma correspondente vontade política. Alguns desses fatores estão na base das dificuldades enfrentadas pela população de Angola para ter acesso à assistência sanitária.

O Comitê Regional Africano/Organização Mundial da Saúde (CRA/OMS) ${ }^{1}$ destaca que, para os países da região, o acesso seria facilitado por meio de serviços distritais de saúde em bom funcionamento, capazes de proporcionar intervenções essenciais às comunidades, famílias e indivíduos em devido tempo e a custo acessível. Contudo, a regionalização dos serviços de saúde, também conhecida por distritalização, entre outras designações, ainda não é levada devidamente em consideração como uma das estratégias 
fundamentais para organização dos sistemas de saúde em muitos países africanos. O mesmo se verifica em Angola, onde, ainda que timidamente, priorizam-se investimentos no nível dos cuidados secundários, o que agrava sobremaneira as dificuldades de acesso e utilização dos serviços de saúde pela população.

A discussão da temática torna-se ainda mais relevante pelo fato de estar em curso em Angola, desde 1999, a implementação de um programa de desconcentração e descentralização administrativas com vista à melhoria do acesso da população aos serviços públicos 2 .

Este artigo tem por objetivo discutir os benefícios, possibilidades e dificuldades para a implementação da regionalização do sistema de serviços de saúde de Angola, considerando seu contexto sócio-sanitário.

\section{Metodologia}

O presente estudo apóia-se numa revisão bibliográfica. Assim, considerando os objetivos traçados, buscaram-se autores brasileiros, uma vez que, no Brasil, está ainda em curso a reforma sanitária, existindo uma vasta produção bibliográfica sobre a temática. Portanto, foram selecionados trabalhos de autores reconhecidos na área.

Com o propósito de identificar estudos e discussões sobre a desconcentração e descentralização das políticas públicas em curso em Angola e também a fim de levantar dados sobre a sua situação sanitária, efetuou-se um deslocamento àquele país, o que permitiu o acesso a publicações sobre esse processo, sobre o seu sistema de saúde e a relatórios estatísticos recentes do Ministério da Saúde de Angola.

Para complementar as informações, foram utilizados documentos do CRA/OMS e de outras organizações das Nações Unidas, como também páginas da internet de instituições da União Européia.

Considerando a necessidade de se analisarem outras experiências regionais e mundiais, recorreu-se a autores africanos ou com estudos sobre o continente, a autores internacionais e a estudos e recomendações da OMS para a Região Africana.

A consulta foi feita nas bibliotecas e bases virtuais, como a SciELO (Scientific Electronic Library Online), a BVS (Biblioteca Virtual em Saúde) e a PAHO (acervo da biblioteca da Organização Pan-Americana da Saúde), além de nas bases de dados da MEDLINE (Literatura Internacional em Ciências da Saúde), LILACS (Literatura Latino-Americana e do Caribe em Ciências da Saúde) e WHOLIS (sistema de informação da bi- blioteca da OMS). A página de buscas da Internet "Google" (http://www.google.com) possibilitou um amplo acesso às mais diversificadas fontes de dados. As palavras-chave utilizadas foram: distritos sanitários, distritalização, municipalização, regionalização, descentralização e acesso em saúde (em Português, Inglês e Francês), de forma isolada e/ou combinada com as palavras "Angola" e "África”. Outra expressão pesquisada foi "situação sócio-sanitária de Angola”. Todo o trabalho de pesquisa e categorização de dados foi realizado de 3 de agosto de 2006 a 2 de dezembro de 2007.

Todos os artigos e documentos passaram por uma leitura em profundidade, orientada pelas categorias: situação de Angola, descentralização, aspectos conceituais, princípios organizativo-assistenciais, benefícios, condições e dificuldades para a regionalização em saúde, ordenados de forma a permitir uma adequada apresentação e abordagem. Foi selecionada principalmente bibliografia recente, dos anos 2000 (Tabela 1).

Foram incluídos todos os trabalhos que se enquadravam na temática, inclusive alguns das décadas de 70 a 90, considerando as características semelhantes dos contextos dos referidos estudos ao de Angola hoje.

Foram excluídos os artigos fora da temática traduzida pelas categorias acima.

\section{Caracterização da situação de Angola}

Situada no Sudoeste de África, conta com 18 províncias, 163 municípios e 475 comunas. Tem aproximadamente $1.246 .700 \mathrm{~km}^{2}$, com uma população estimada em 15.107.000 habitantes 3, encontrando-se cerca de $49 \%$ ainda concentrada nos grandes centros urbanos, resultado da guerra que o país enfrentou desde a independência, em 1975 , até 2002. A taxa de alfabetização é de $42 \%$, segundo o Instituto Português de Apoio ao Desenvolvimento (IPAD) 4. Além da língua oficial, o Português, existem no país vários grupos etnolingüísticos, expressando-se em mais de vinte línguas nacionais, línguas maternas da maioria da população ${ }^{5}$.

\section{Perfil político-administrativo}

A Constituição estabelece um sistema político semipresidencial. No nível subnacional, o poder está concentrado nos governos de província e nos seus governadores, nomeados pelo Presidente da República. No entanto, com a descentralização de responsabilidades administrativas, do nível central para o provincial em curso, o setor da saúde também vive uma descentralização par- 
Bibliografia selecionada.

\begin{tabular}{|c|c|c|c|c|c|c|c|}
\hline \multirow[t]{2}{*}{ Origem } & \multicolumn{2}{|c|}{ Artigos/Capítulos } & \multirow[t]{2}{*}{ Manuais } & \multirow[t]{2}{*}{ Livros } & \multirow{2}{*}{$\begin{array}{l}\text { Páginas da } \\
\text { Internet }\end{array}$} & \multirow{2}{*}{$\begin{array}{l}\text { Documentos do CRA/ } \\
\text { OMS, PNUD, MAT, } \\
\text { MINSA, OMS, INLCS, } \\
\text { dissertações ou teses }\end{array}$} & \multirow[t]{2}{*}{ Total } \\
\hline & Revistas & Livros & & & & & \\
\hline Brasileiros & 7 & 2 & 1 & 6 & 1 & - & 17 \\
\hline Africanos ou sobre África & 3 & - & 1 & - & - & - & 4 \\
\hline Estudos e dados sobre Angola & - & 1 & - & 2 & 4 & 6 & 13 \\
\hline Internacionais & - & - & - & 1 & - & 2 & 3 \\
\hline Total & 10 & 3 & 2 & 9 & 5 & 8 & 37 \\
\hline
\end{tabular}

CRA/OMS: Comitê Regional Africano/Organização Mundial da Saúde; PNUD: Programa das Nações Unidas para o Desenvolvimento; MAT: Ministério da Administração do Território; MINSA: Ministério da Saúde de Angola; INLCS: Instituto Nacional de Luta Contra a Sida de Angola.

cial de autoridade e de orçamento, do Ministério da Saúde de Angola para hospitais de cuidados terciários e provinciais e institutos 6 .

\section{Perfil epidemiológico}

As principais endemias são a malária, que representa $50 \%$ da demanda; a tuberculose; o HIV/ AIDS; a tripanossomíase africana; a lepra e a esquistossomose 7 , confrontando-se o país com altas taxas de mortalidade infantil (154/mil nascidos vivos) 4 , sendo a de menores de cinco anos na ordem de $250 /$ mil nascidos vivos 3 . A mortalidade materna está em torno de 1.500 por 100 mil nascidos vivos. A geral apresenta-se bastante elevada, $18,8 \%$, para uma taxa de natalidade de $48,4 \% 4$

Estimativas do Instituto Nacional de Estatística (INE) para o ano de 2003, segundo o Instituto Nacional de Luta Contra a SIDA (INLCS) ${ }^{8}$, indicam uma cobertura dos serviços de atenção pré-natal de $66 \%$, uma taxa de utilização inferior a $40 \%$, limitado acesso aos serviços de saúde, avaliado em $50 \%$, e somente $45 \%$ dos partos são atendidos por profissionais em unidades de saúde.

\section{Recursos humanos}

Angola tem cerca 1.458 médicos (nacionais e estrangeiros) 9 , estando mais de $70 \%$ concentrados na capital, Luanda, e seus arredores, com cerca de 5 milhões de habitantes, um terço da população angolana; em geral, o país tem 0,64 médico nacional para cada 10 mil habitantes 9 . Observa-se também uma elevada concentração de enfermeiros e de outros profissionais da saúde em Luanda, sendo o coeficiente nacional de
12,19 enfermeiros por 10 mil habitantes 9 , inviabilizando o reforço das redes locais de saúde e a intensificação de intervenções prioritárias de saúde que respondam às necessidades das comunidades locais.

Em geral, verifica-se uma profunda assimetria na distribuição espacial dos servidores civis do Estado, particularmente com os subuniversos do pessoal técnico superior e do técnico: $72 \%$ do primeiro e $81 \%$ do segundo encontramse na capital, concluindo-se que é profunda a fraqueza institucional na maioria das províncias de Angola 10.

\section{Financiamento da saúde}

No período de 1997 a 2001, a despesa do Estado com a saúde foi de $3,3 \%$ do produto interno bruto (PIB), enquanto a média dos países da Comunidade de Desenvolvimento da África Austral (SADC) foi de $7,2 \%$. Do total, mais de $50 \%$ foram destinados ao nível secundário e a unidades centrais 6 .

\section{Rede de saúde}

Parte encontra-se ainda degradada, como conseqüência da guerra, baixo investimento em construção e manutenção, em especial nas zonas periféricas, registrando-se uma proporção de 0,77 cama para mil habitantes e de 49.852 habitantes por centro de saúde 10 .

O sistema de saúde de Angola reflete uma estrutura organizacional essencialmente curativa, ainda precária, sem adequado desenvolvimento do processo de descentralização e do sistema de referência em que os distritos, ou pelo menos os municípios, sejam unidades de importância 
fundamental. Essa situação explica a escassez de estruturas intermediárias - hospitais provinciais e municipais - e periféricas - centros e postos de saúde 11. Assim, a inexistência de um sistema de referência e contra-referência operacional é uma das dificuldades do sistema de saúde.

\section{Regionalização: contexto e desafios para o caso de Angola}

De acordo com o exposto, pode-se concluir que o sistema de saúde de Angola apresenta enormes deficiências de estruturação e funcionamento, o que leva à baixa resolubilidade dos seus serviços. Vemba 12, em seu estudo As Três Dimensões Críticas do Sistema de Saúde Angolano, também conclui que este sistema não satisfaz as exigências da realidade atual, sendo, por isso, necessária a sua total reformulação.

Assim, para a inversão da situação, considera-se importante um grande investimento para (re)construção e reestruturação da rede, para formação e contratação de recursos humanos e para aquisição de insumos e tecnologias; a regulamentação e a introdução de reformas que dêem continuidade à descentralização culminante com a regionalização dos serviços de saúde, entre outras ações. Entre elas, destaca-se a revitalização dos cuidados primários de saúde, o que proporcionaria um bom enquadramento para o acesso universal aos cuidados essenciais de saúde ${ }^{1}$.

No entanto, a modalidade de descentralização ocorrida até aqui em Angola, no setor da saúde, segundo vários autores 13,14,15, é designada por desconcentração que sugere a transmissão de certas responsabilidades e funções, sem a transferência correspondente de poder decisório. Para Silveira 15, descentralização significa redistribuir as responsabilidades quanto às ações e aos serviços de saúde entre os vários níveis do governo, partindo da idéia de que quanto mais perto do fato a decisão for tomada, mais chances haverá de acerto. A autora afirma que não se deve confundir com desconcentração ou delegação, pois descentralização envolve compartilhamento do poder. Deve haver uma profunda redefinição das atribuições dos vários níveis do governo com o nítido reforço do poder municipal sobre a saúde, cabendo ao município, portanto, a maior responsabilidade das ações e a aproximação do usuário do poder decisório do sistema.

A descentralização tornou-se, a partir de 1999, tema central da agenda pública e institucional do governo de Angola. Adiada ao longo de anos, a questão parece ter, definitivamente, ganho espaço na agenda institucional do país, particularmente depois da aprovação do programa do governo para 2003-2004 2. Segundo a fonte, a descentralização pode ser um mecanismo institucional de reforço e consolidação da unidade nacional, coesão social e de promoção da democracia local, como também uma resposta adequada à diversidade étnica e geográfica de Angola. Assim, devem ser transferidas da administração central para a administração local do Estado designadamente funções nos domínios da saúde, terras, agricultura, habitação, obras públicas e urbanismo, educação, comércio e transportes.

Entretanto, as especificidades do setor da saúde devem ser consideradas no processo em curso, pois o modo de organizar as redes de atenção à saúde define a singularidade de seus processos descentralizadores frente a outros setores sociais 16. Os serviços de saúde estruturam-se numa rede de pontos de atenção à saúde composta por equipamentos de diferentes densidades tecnológicas que devem ser distribuídos espacialmente, de forma ótima. Essa distribuição ótima vai resultar em eficiência, efetividade e qualidade dos serviços. É nesta base que os principais objetivos da OMS 17 para Angola são justamente o apoio à reabilitação do sistema de saúde municipal, de modo a melhorar a prestação de serviços de saúde de qualidade nos níveis nacional, provincial e municipal, e a promoção de meios de subsistência sustentáveis, boa governança e descentralização.

Para Campos 18, o horizonte da descentralização é o município. Contudo, para a maioria dos municípios, é impossível compor no próprio território toda a complexa rede de serviços e prestações de saúde necessários para assegurar acesso e atenção conforme cada caso ${ }^{18}$. O mesmo também se poderia dizer quanto aos municípios e algumas províncias de Angola com as carências financeiras vivenciadas e com os constrangimentos que obliteram as capacidades locais do exercício da função governativa, nomeadamente, nível reduzido dos salários, baixa produtividade administrativa, recursos financeiros limitados, deficiente capacidade institucional, déficit de informação para a decisão, gap de recursos humanos qualificados, entre outros 10 .

A hierarquização de serviços, ou seja, a construção de uma rede básica com ampla cobertura populacional e capacidade para resolver problemas clínicos e de saúde pública, apoiada por uma variada rede de serviços especializados - de urgência, hospitalares e ambulatoriais -, é um modo de organizar sistemas de saúde que não só assegurem o acesso à atenção e a integralidade desta, como também dêem viabilidade financeira a essas políticas públicas. Seria 
impossível e irracional instalar em cada município todos os serviços especializados de saúde. A criação de regiões de saúde é, pois, condição sine qua non para a constuição de um sistema público de saúde 18 que seja acessível para a população, incluindo a mais necessitada.

A descentralização como um processo social, na prática, apresentará, sempre, fortalezas e debilidades, mas a questão central está em desenvolver processos de descentralização dos sistemas de serviços de saúde que maximizem as suas fortalezas e minimizem as suas debilidades. Nesse sentido, uma das propostas mais consistentes está em instituir, entre os níveis centrais e locais dos sistemas de serviços de saúde, uma instância mesorregional, sejam distritos sanitários ou regiões de saúde 14. Esses argumentos sustentam a necessidade e a importância da proposta da regionalização dos sistemas de saúde.

Consideramos a regionalização como uma das estratégias para (re)organização da rede de serviços e como um dos instrumentos para a (re)construção de um sistema de saúde integrado de Angola. É um esquema de implantação racional dos recursos de saúde, organizados dentro de uma disposição hierárquica, na qual seja possível oferecer uma assistência máxima nos centros primários, sendo os demais serviços utilizados apropriadamente de acordo com as necessidades individuais do paciente 19. Dentre os princípios organizativo-assistenciais do distrito sanitário 20 , capazes de conferir a qualidade exigida para tornar-se uma opção para responder aos problemas e necessidades de saúde da realidade local, para além da hierarquização, destacam-se, a orientação por problemas, a intersetorialidade, o planejamento e a programação local. Outros princípios são a intercomplementaridade, a integralidade, a adscrição, a heterogeneidade e a autoridade sanitária local a ser conseguida através da descentralização. Porém, um Sistema Distrital de Saúde só pode funcionar plenamente após implementação da descentralização, devendo ter regulamentação e legislação próprias 21 .

Um sistema de saúde descentralizado, em uma dinâmica de transferência de recursos e autoridade às suas diferentes instâncias, causa impacto positivo na gestão e nas diferentes modalidades de atenção. Em adição, dá oportunidade para que os processos de reforma permitam a geração e desenho de novos modelos de atenção, papéis e funções, modalidades de capacitação, sistemas de remuneração e novas formas de participação das instituições, incluindo setores acadêmicos 22 .

O princípio da hierarquização está determinado pela garantia de resolubilidade que se deve dar de acordo com a complexidade tecnológica exigida em cada nível e no nível do sistema como um todo. Por conseguinte, a visão dialética da descentralização prevê que a cada movimento de descentralização corresponde, até certo ponto, uma contraposição de centralização relativa. Assim, se a atenção primária deve necessariamente ser prestada desconcentrada e descentralizadamente, os níveis secundário e terciário organizam-se de forma concentrada e centralizada 14 . Portanto, a regionalização implica necessariamente que "os serviços com menor grau de especialização achem-se espacialmente distribuídos e os mais especializados estejam mais centralizados" 19 (p. 43).

O sistema integrado de transferência do paciente por sucessivos níveis de atenção está baseado na premissa de que os hospitais são instituições com cuidados especializados e que seus custos são substancialmente reduzidos através da implantação de outros serviços periféricos mais simplificados e resolutivos. A disponibilidade de ambulatórios e serviços domiciliares teria um efeito significativo não tanto sobre a taxa de hospitalização, mas sobre a permanência hospitalar 23 .

Defendemos para Angola a descentralização dos cuidados primários, cuja rede inclui os postos de saúde, dispensários e centros de saúde com ou sem camas, e a regionalização dos serviços de maior densidade tecnológica, como hospitais, atenção ambulatorial especializada e serviços de apoio diagnóstico e terapêutico de referência, mediante a criação de regiões e microrregiões ou áreas sanitárias. No processo de construção destes espaços territoriais sanitários, há a necessidade de se criarem e implantarem instrumentos e mecanismos que garantam a articulação entre os serviços de saúde dos distintos níveis e diferentes municípios e bairros, num processo dinâmico e flexível denominado referência e contra-referência 24 , considerado fundamental para se atingir a eqüidade, qualidade e integralidade do cuidado. Concomitantemente, há que se pensar não só em estruturas de apoio, como vias de acesso e comunicação, meios de transporte, como também em operações de remoção de minas terrestres, uma vez que a existência desses artefatos é um nefasto legado da guerra naquele país, entre outras.

Todavia, para uma exitosa regionalização em saúde, há necessidade de considerar as seguintes condições 19: (a) informação demográfica e epidemiológica da população objeto; (b) realização de um inventário atualizado dos serviços existentes; (c) informação sobre a utilização dos recursos; (d) consenso comunitário sobre as prioridades; (e) conhecimento dos diversos aspectos da acessibilidade aos serviços para corrigir as principais deficiências do sistema e de 
sua prestação; (f) relação entre formação, pelas entidades educadoras, de graduados nas ciências de saúde e oportunidades de emprego; (g) novos mecanismos de cooperação intersetorial com planejamento conjunto, controle de qualidade e a avaliação dos serviços.

As condições supracitadas, mesmo não satisfeitas a priori, devem ser vistas de forma dinâmica no próprio processo de implementação da estratégia da regionalização.

A falta de dados atualizados ou o fato de os dados disponíveis serem estimados e alguns pouco confiáveis, ocultando questões potencialmente graves ou ocasionando distorções e erros de interpretação, representam duas das grandes dificuldades para o caso de Angola. Assim, ambos deveriam ser o foco das atenções dos formuladores e implementadores da estratégia, pois a informação estratégica sobre a situação em análise é fundamental por ajudar na formulação correta das ações a serem implementadas e por facilitar o consenso, embora esta não seja uma dimensão fácil. Por tratar-se de um processo com várias etapas para a sua implementação, muitas das condições poderão ser paulatinamente atingidas mediante a interação entre formuladores, implementadores e o público-alvo.

Artmann \& Rivera 25 chamam a atenção para o fato de que a reestruturação dos serviços de saúde pela via da regionalização é um processo de busca permanente da negociação e da cooperação, que exige flexibilidade, não passível de ser resolvido pela aplicação de um instrumento normativo homogêneo. Entretanto, em Angola, não existe uma tradição de participação dos órgãos locais ou de base nos processos de decisão dos governos provinciais ou de administração central. Não há cultura de participação dos atores sociais nem de negociação ${ }^{2}$, o que exige que a descentralização/regionalização naquele país inclua estas dimensões como processo de aprendizagem 26.

A cooperação é essencial, tendo em vista que, com a regionalização, propõe-se encontrar um equilíbrio entre a excessiva centralização estrutural e a descentralização total dos serviços de saúde 26 . Nesse sentido, a experiência internacional indica que existem áreas em que a descentralização não deve incidir: na definição de macro-políticas de saúde, nas decisões referentes aos recursos estratégicos, na regulação de setores como de medicamentos e de equipamentos de alta densidade tecnológica e na macro-análise de situação e tendências de saúde 14 .

A experiência de Quebec (Canadá) e da França 25 demonstra que a dinâmica de mudança, como a que mencionamos neste trabalho, depende de vários indivíduos em diferentes instâncias e esferas de poder, situação que coloca em pauta a noção de liderança coletiva, pois "não supõe apenas alargar a capacidade de decisão dos dirigentes locais e regionais, mas a necessidade de articular várias lideranças, situadas em diversas esferas de poder e níveis do sistema" 27 (p. 1).

Logo, com a regionalização, a responsabilidade pela administração do sistema de saúde de Angola ficaria distribuída pelos níveis nacional, provincial e municipal; no último, algumas atribuições seriam compartilhadas com as autoridades comunais. No entanto, a identificação das linhas de intervenção e a conformação dos sistemas funcionais de saúde não seriam necessariamente restritas à abrangência da região político-administrativa (província, município ou comuna), mas respeitariam seus limites como unidade indivisível, objetivando garantir a integralidade da assistência e o acesso da população aos serviços e ações de saúde consoante as necessidades. Surgiriam, assim, as regiões funcional-assistenciais, pois as ações correspondentes se dão em territórios concretos, onde as pessoas vivem, trabalham, constroem redes de relacionamento e exercitam o poder 28 . Só é possível articular as diversas políticas públicas de nível setorial com incidência provincial e as políticas de âmbito nacional, provincial e local 10 considerando-se o território.

Em Angola, por ser um Estado unitário e com uma dimensão territorial não muito extensa, de acordo com as especificidades geográficas, demográficas, epidemiológicas, econômicas, as províncias e os municípios de grande porte poderiam ser agrupados para a conformação das regiões ou distritos sanitários, enquanto os municípios de menor porte, comunas e/ou bairros, por sua vez, para a conformação das microrregiões ou áreas sanitárias.

Dentro de um sistema de serviços regionalizados 23, a distância seria um dos critérios mais importantes para estabelecer a distribuição geográfica das instituições de saúde. Em um país com grandes distâncias, diferenças regionais enormes, destacando-se os extremos entre regiões quase despovoadas e centros urbanos superpopulosos, é preciso pensar com criatividade e contextualizar os parâmetros através de adaptações viáveis, que tragam impactos positivos sobre a situação problemática. Essa formulação cabe perfeitamente para o caso de Angola, que vive, em adição, problemas de comunicação.

Um estudo feito na Zâmbia 29 concluiu que aproximadamente $50 \%$ das pessoas que vivem a menos de $5 \mathrm{~km}$ de um provedor de saúde decidem procurar assistência quando se sentem mal, e somente $17 \%$ daquelas que vivem a mais de $40 \mathrm{~km}$ tomam essa mesma decisão. Essa con- 
clusão suporta a noção de que o grau de acesso real aos serviços de saúde depende da distância que se deve percorrer para obtê-los, do tempo de viagem e do seu custo ${ }^{30}$. As especificidades de Angola, no concernente à circulação de pessoas e bens, deveriam ser levadas em consideração na formulação das políticas para o setor da saúde.

Onokerhoraye 31 sugere, na Nigéria, uma distribuição de serviços de saúde segundo a qual os centros de saúde das comunidades deveriam servir a uma população de $10 \mathrm{mil} \mathrm{a} 20 \mathrm{mil}$ pessoas. Nesses centros, a área de influência é aquela em que o usuário tem de percorrer, no máximo, um quilômetro para chegar à unidade de saúde mais próxima. Este e outros estudos feitos em África, alguns relativamente antigos, mas já bem testados na prática, em virtude da proximidade do contexto, podem servir de referência, desde que os seus resultados sejam adaptados às especificidades locais. Recomenda-se que o estabelecimento das referidas áreas de influência e a definição dos padrões médico-assistenciais sejam adaptados a cada contexto e à realidade de cada região e país 32,33 .

A regionalização da saúde em Angola é um grande desafio, não só pela tradição de centralização, mas também pelos grandes vazios assistenciais existentes. Contudo, constitui-se numa importante estratégia, pois facilitaria a discussão sobre o ajuste da oferta de serviços de saúde, diminuiria a pressão da demanda por serviços localizados nos grandes centros urbanos com a redefinição da porta de entrada para o sistema, propiciaria mecanismos para a superação da fragmentação dos serviços e para instituição de um sistema integrado de saúde 14 . Ademais, contribuiria para criar consciência de pertencimento à região sanitária, fortaleceria a cooperação institucional, ofereceria um mecanismo mais eficiente para a distribuição de recursos humanos e equipamentos entre todos os estabelecimentos, inter-relacionando o central e o periférico, a fim de facilitar a referência de pacientes, fluxo de registros médicos e a consulta aos médicos especialistas por parte de generalistas 19 .

Realizada num processo participativo, poderia promover maior aceitação e conhecimento dos serviços por parte da população, acesso pronto dos usuários a serviços de qualidade ao menor custo social e econômico possível, bem como proporcionar a constituição de mecanismos mais adequados de supervisão e o controle 16,28. Com a regionalização, as autoridades locais poderiam determinar as prioridades concernentes à prestação de serviços de saúde na sua área de jurisdição e alocar recursos de acordo com essas prioridades 15,34.
Finalmente, com a estratégia de regionalização, as ações de vigilância, educação, atenção ambulatorial e hospitalar seriam favorecidas 15 . Os serviços de atenção primária responderiam melhor às necessidades dos usuários 28 , aumentando, assim, a resolubilidade do sistema de saúde, desde que houvesse um investimento na rede física e em recursos humanos e tecnológicos.

Apesar de todos esses benefícios, os esforços para regionalizar um sistema de saúde trazem em si um grande peso político, já que essas soluções são de médio e longo prazo, e os líderes políticos procuram sempre respostas em prazos muito mais curtos. Na ausência de um plano educacional para os provedores, pessoal de saúde e o próprio público, o conceito pode antagonizar todos esses elementos, impedindo sua implementação (Fein, 1982, apud Novaes 19). É importante sublinhar que a regionalização de um sistema de saúde com organização hierárquica da rede é bastante complexa, impondo grandes desafios administrativos relacionados a diferentes processos, tais como negociação e geração de consensos, estabelecimento de regras de atuação, distribuição de recursos e interação, construção de mecanismos decisórios coletivos, estabelecimento de prioridades e acompanhamento 35. Uma legislação apropriada é imprescindível para permitir o estabelecimento de uma administração sólida e uma coordenação eficiente. Considera-se também crucial para um bom funcionamento dos serviços distritais de saúde, para o nível local, a autonomia financeira, a responsabilidade de recrutamento de pessoal e desenvolvimento de adequadas infra-estruturas e capacidades técnicas 36 . Finalmente, as decisões políticas devem ser orientadas para criação da estrutura necessária para o efeito desejado ${ }^{21}$.

Tomando como base autores aqui trabalhados e o contexto apresentado, para a formulação e, sobretudo, para a implementação da regionalização de saúde em Angola, podem surgir várias dificuldades, considerando-se que são necessárias mudanças substanciais na organização e funcionamento dos sistemas de saúde, pressupondo operações de grande complexidade técnica, política, logística, administrativa e comunicativa. Apresentamos, a seguir, as que se nos afiguram mais preocupantes:

a) Déficit de profissionais, destacando-se também as distorções na sua distribuição, com uma grande concentração nos grandes centros urbanos e em cinco províncias do litoral, onde se encontram, por exemplo, 85\% dos médicos do país 10 . A grande dificuldade consistiria na falta de profissionais para a periferia.

b) Não percepção imediata pelo público da lógica de regionalização da assistência, o que pode 
provocar incompreensões e resistências nas fases iniciais da implantação da estratégia, exigindo, portanto, paralelamente, um grande trabalho de informação e educação da população.

c) Rede sanitária deficiente e/ou insuficiente, demandando grande investimento para o alargamento da rede nos três níveis de atenção, particularmente para o nível secundário.

d) Baixa qualificação do pessoal administrativo e técnico, falta de atualização dos seus conhecimentos, desconhecimento de novos conceitos de organização e gestão dos serviços de saúde. Exigir-se-ia um grande investimento na formação do pessoal.

e) Baixa motivação dos trabalhadores da saúde, em virtude dos baixos salários e da falta de melhores incentivos, o que dificultaria a colocação e fixação desses profissionais nas áreas do interior.

f) A realidade sócio-cultural de certas regiões e a previsível interferência de atores com outros interesses e visões podem influenciar negativamente os grupos técnicos de trabalho, conduzindo-os à adoção, na prática, de uma conduta que não corresponda integralmente com anseios de ordem técnica.

g) Deficiente rede de transporte, comunicações e de outras infra-estruturas básicas, o que dificultaria a circulação de pessoas (técnicos e usuários) e bens.

h) Dificuldades na definição das macrorregiões e microrregiões, em face do atraso e/ou desequilíbrio no desenvolvimento das províncias, municípios e comunas, fazendo com que se verificassem falhas na sua vinculação aos centros a serem definidos.

i) Insuficiente e/ou deficiente estrutura por parte das administrações locais para gerir seus serviços e subsistemas de saúde, o que poderia requerer uma assessoria externa e uma grande capacidade de supervisão dos níveis hierarquicamente acima.

j) Déficit e/ou falta de dados confiáveis que pudessem ajudar na formulação das ações, podendo conduzir a erros na definição de ações e, como já mencionado, dificultar o alcance de consensos, algo fundamental para implantação da estratégia.

l) Insuficientes recursos financeiros e o fraco desenvolvimento de capacidades gestoras nos distintos níveis que compõem o sistema. A despesa financeira 6 do Estado angolano com a saúde, de 3,3\% do PIB, é manifestamente ínfima até em comparação com a média dos países da sub-região, e os $10 \%$ do referido montante para investimento não correspondem com os propósitos de uma reforma da saúde que exige uma grande aplicação de recursos inicial.
Independentemente das dificuldades enumeradas, a regionalização é uma tendência universal e irreversível 2. É vista como solução adequada onde os recursos são escassos e os problemas, complexos 35 , e onde há uma crescente demanda por serviços e ações de saúde, como é o caso de Angola.

Considerando as necessidades assistenciais verificadas naquele país, resultantes de infra-estrutura e financiamento insuficientes, concomitante à implementação da estratégia de regionalização, que representa uma política racionalizadora, devem ser feitos grandes investimentos na rede, visando à integração de ações de promoção, prevenção, assistência curativa e reabilitação. Para tanto, a vontade política é fundamental para a captação de recursos necessários para reconstrução da rede, aquisição de meios técnicos, formação de pessoal, importantes elementos para reorganização e gestão estratégica de um sistema de serviços de saúde.

Todos os 46 países da região africana da OMS iniciaram um tipo de reforma do setor da saúde, variando de um país a outro os contextos e os indicadores dos programas da reforma, a qual foi influenciada, na maior parte, pelo fraco desempenho dos seus sistemas da saúde, particularmente no que diz respeito à qualidade de serviços de saúde 37 . Os processos em causa têm como bases fundamentais a descentralização, a municipalização e a criação de distritos sanitários.

Apesar do ainda fraco desempenho dos sistemas de saúde de África, a implementação gradual da regionalização, que considere as realidades e circunstâncias político-administrativas, epidemiológicas, econômicas, culturais, gerenciais, cognitivas locais, de acordo com as experiências analisadas, incluindo de países africanos 36 , poderia representar uma adequada estratégia para a melhoria do acesso aos cuidados de saúde, particularmente para os grupos economicamente menos favorecidos. Em adição, poderia potencializar o processo de descentralização 28 em curso.

A diversidade cultural e étnico-lingüística são fatores a serem levados em conta, sem perder de vista a necessidade da contínua integração dos povos de Angola. Uma estratégia interessante de enfretamento das dificuldades pode ser o recurso a assessorias externas, como a OMS, organizações não-governamentais (ONGs) e instituições de pesquisa científica. Contudo, a avaliação do seu impacto só poderá ser feita a longo prazo, analisando-se o grau de melhoria ou não do acesso da população aos cuidados de saúde. 


\section{Resumo}

Este artigo apresenta a situação da saúde de Angola no período 2000-2007, discute a regionalização do sistema de saúde como importante instrumento para a melhoria tanto do acesso aos serviços de saúde daquele país, quanto da utilização destes, e aponta condições e desafios para a sua implementação. $O$ artigo se apóia numa revisão bibliográfica de: (a) autores brasileiros, considerando-se que, no Brasil, ainda decorre a reforma sanitária com vasta produção bibliográfica sobre a temática; (b) autores africanos, ou com estudos sobre a África, e internacionais, em vista da necessidade de se analisarem experiências regionais e mundiais relativas ao assunto e (c) estudos e recomendações da Organização Mundial da Saúde sobre a descentralização/regionalização em saúde. Ficou demonstrada a necessidade de reformas no sistema de saúde de Angola, o qual apresenta enormes deficiências de estruturação e funcionamento, o que leva à baixa resolubilidade dos seus serviços. A regionalização foi considerada como uma importante estratégia para reestruturação do sistema de saúde, ao lado de outras que não são foco principal deste estudo. A avaliação do seu impacto só poderá ser feita a longo prazo.

Acesso aos Serviços de Saúde; Regionalização; Sistemas de Saúde

\section{Referências}

1. Comitê Regional Africano, Organização Mundial da Saúde. Revitalização dos sistemas de saúde no contexto dos cuidados primários de saúde na região africana, 2006. http://afrolib.afro.who.int/ RC/RC\%2056/Doc_Por/AFR\%20RC56\%2012.pdf (acessado em 20/Ago/2006).

2. Ministério da Administração do Território/Programa das Nações Unidas para o Desenvolvimento. Desconcentração e descentralização em Angola. v. II. Luanda: Ponto UM. Indústria Gráfica; 2007.

3. Fundo de População das Nações Unidas. Características gerais da população de Angola, 2006. http://www.angola.unfpa.org/popang.htm (acessado em 04/Dez/2006).

4. Instituto Português de Apoio ao Desenvolvimento. Angola. http://www.ipad.mne.gov.pt/index. php?option=com_content\&task=view\&id=29\&Ite mid=60 (acessado em 16/Jul/2007).

5. Menezes S. Mamma Angola: sociedade e economia de um país nascente. São Paulo: Edusp; 2000.

6. Organização Mundial da Saúde. Estratégia da OMS de cooperação com os países: Angola, 2002-2005. http://www.who.int/countryfocus/cooperation strategy/countries/angola_2002_2005_portuguese.pdf (acessado em 14/Out/2006).

\section{Colaboradores}

M. S. Oliveira participou do levantamento da literatura e de dados, revisão bibliográfica, análise dos dados e redação do artigo. E. Artmann colaborou na orientação metodológica, desenho da pesquisa e redação do artigo.

7. Bungo F. Estudo da prevalência da filariose bancroftiana e loana na Vila do Buco-Zau, Norte de Angola [Dissertação de Mestrado]. Rio de Janeiro: Escola Nacional de Saúde Pública, Fundação Oswaldo Cruz; 2002.

8. Instituto Nacional de Luta Contra a SIDA de Angola/Joint United Nations Programme on HIV/AIDS. Relatório de UNGASS 2006. http://data.unaids. org/pub/Report/2006/2006_country_progress_ report_angola_pt.pdf (acessado em 08/Set/2007).

9. Cassoma B, Vinyals L. Angola: despesa pública no setor da saúde (2000-2007). Estoril: Principia Editora; 2007.

10. Fonseca PL. Preparação e organização das administrações locais para a implementação do programa do governo e acções complementares. In: Encontro Nacional sobre Administração Local em Angola. Luanda: Editorial Nzila; 2005. p. 99-113.

11. Campione A. Análise dos principais recursos na área de saúde presentes na Província de Luanda. Luanda: Centro Interuniversitário de Investigação dos Países em Desenvolvimento, Programa de Vigilância Epidemiológica em Angola, Cooperação Itália-Angola; 1998. 
12. Vemba LV. As três dimensões críticas do sistema de saúde angolano, 1997-2002. Luanda: United States Agency for International Development; 2002.

13. Pasche DF, Righi LB, Thomé HI, Stolz ED. Paradoxos das políticas de descentralização de saúde no Brasil. Rev Panam Salud Pública 2006; 20:416-22.

14. Mendes EV. Os grandes dilemas do SUS. Salvador: Casa da Qualidade Editora; 2001. (Saúde Coletiva, 4).

15. Silveira $\mathrm{CH}$. Algumas considerações a respeito das políticas de saúde no Brasil. In: Battaglin P, Leandro AL, Michaliszyn MS, organizadores. Saúde coletiva: um campo em construção. Curitiba: Instituto Brasileiro de Pós-Graduação e Extensão; 2006. p. 29-69.

16. Pestana M, Mendes EV. Pacto de gestão: da municipalização autárquica à regionalização cooperativa. Belo Horizonte: Secretaria de Estado de Saúde Minas Gerais; 2004.

17. Organização Mundial da Saúde. Angola: contexto, 2006. http://www.unangola.org/oms.asp (acessado em 02/Fev/2007).

18. Campos GWS. Efeitos paradoxais da descentralização no Sistema Único de Saúde do Brasil. In: Fleury S, organizadora. Democracia, descentralização e desenvolvimento: Brasil \& Espanha. Rio de Janeiro: Editora FGV; 2006. p. 417-42.

19. Novaes HM. Ações integradas nos Sistemas Locais de Saúde - SILOS: análise conceitual e apreciação de programas selecionados na América Latina. São Paulo: Livraria Pioneira Editora; 1990.

20. Mendes EV. Distrito sanitário: o processo social de mudança das práticas sanitárias do Sistema Único de Saúde. São Paulo: Editora Hucitec/Rio de Janeiro: ABRASCO; 1993.

21. Chatora R, Tumusiime P. Health sector reform and district health systems. http://www.afro.who.int/ dsd/dhm-training/mod1.pdf (acessado em 10/ Nov/2007).

22. Hortale VA, Pedroza M, Rosa MLG. Operacionalizando as categorias acesso e descentralização na análise de sistemas de saúde. Cad Saúde Pública 2000; 16:231-9

23. Artmann E, Rivera FJU. Regionalização em saúde e mix público-privado. http://www.ans.gov. br/portal/upload/biblioteca/TT_AS_05_EArt mann_RegionalizacaoEmSaude.pdf (acessado em 06/Out/2006).

24. Almeida ES, Castro CGJ, Lisboa CA. Distritos sanitários: concepção e organização. v. 1. São Paulo: Faculdade de Saúde Pública, Universidade de São Paulo; 1998.
25. Artmann E, Rivera FJU. L'hôpital en restructuration: regards croisés sur la France et le Québec [RESENHA]. Cad Saúde Pública 2006; 22:2253-5.

26. Rivera FJU, Artmann E. A liderança como intersubjetividade lingüística. Interface Comun Saúde Educ 2006; 10:411-26.

27. Contandriopoulos D, Contandriopoulos AP, Denis JL, Valette A, editors. L'hôpital en restructuration: regards croisés sur la France et le Québec. Montreal: Les Presses de l'Université de Montreal, 2005

28. Gil AC, Licht RHG, Santos BRM. Regionalização da saúde e consciência regional. Hygeia 2006; 2: 35-46.

29. Hjortsberg CA, Mwikisa CN. Cost of access to health services in Zambia. Health Policy Plan 2002; 17:71-7.

30. Unglert CVS, Rosenburg CP, Junqueira CB. Acesso aos serviços de saúde: uma abordagem de geografia em saúde pública. Rev Saúde Pública 1987; 21:439-46.

31. Onokerhoraye AG. A suggested framework for the provision of health facilities in Nigeria. Soc Sci Med 1976; 10:565-70.

32. Organización Mundial de la Salud. Preparación de indicadores para vigilar los progresos realizados en el logro de la salud para todos en el año 2000. Geneva: Organización Mundial de la Salud; 1981. (Serie Salud para Todos, 4).

33. Organização Pan-Americana da Saúde. Leitos por habitante e médicos por habitante (atualizado em março de 2003). http:/ /www.opas.org.br/sistema/ fotos/leitos.pdf (acessado em 02/Mar/2007).

34. Araújo JD, Ferreira ESM, Nery GC. Regionalização dos serviços de saúde pública: a experiência do Estado da Bahia, Brasil. Rev Saúde Pública 1973; 7:1-19.

35. Fleury S, Ouverney A. Gestão de redes: a estratégia de regionalização da política de saúde. Rio de Janeiro: Editora FGV; 2007.

36. Görgen H, Kirsch-Woik T, Schmidt-Ehry B. Le système de santé de district: experiences et perspectives en Afrique. Manuel à l'intention des professionnels de la santé publique. Eschborn: Deutsche Gesellschaft für Technische Zusammenarbeit (GTZ) GmbH; 2004

37. Lambo E, Sambo LG. Health sector reform in sub Saharan Africa: a synthesis of country experiences. East Afr Med J 2003; 80(6 Suppl):S1-20.

Recebido em 18/Jun/2008 Versão final reapresentada em 06/Out/2008 Aprovado em 04/Nov/2008 